\title{
A galaxy dynamo by Supernova-driven interstellar turbulence
}

\author{
Oliver Gressel, Udo Ziegler, Detlef Elstner and Günther Rüdiger \\ Astrophysikalisches Institut Potsdam, An der Sternwarte 16, 14482 Potsdam, Germany \\ email: ogressel,uziegler,elstner,gruediger@aip.de
}

\begin{abstract}
Supernovae are the dominant energy source for driving turbulence within the interstellar plasma. Until recently, their effects on magnetic field amplification in disk galaxies remained a matter of speculation. By means of self-consistent simulations of supernova-driven turbulence, we find an exponential amplification of the mean magnetic field on timescales of a few hundred million years. The robustness of the observed fast dynamo is checked at different magnetic Reynolds numbers, and we find sustained dynamo action at moderate Rm. This indicates that the mechanism might indeed be of relevance for the real ISM.

Sensing the flow via passive tracer fields, we infer that SNe produce a turbulent $\alpha$ effect which is consistent with the predictions of quasilinear theory. To lay a foundation for global mean-field models, we aim to explore the scaling of the dynamo tensors with respect to the key parameters of our simulations. Here we give a first account on the variation with the supernova rate.
\end{abstract}

Keywords. Turbulence - ISM: supernova remnants - turbulence - magnetic fields

We here present new results on our local box simulations of a differentially rotating, vertically stratified, turbulent interstellar medium threaded by weak magnetic fields (Gressel et al. 2008a). In our model, we apply optically thin radiative cooling and heating to account for the heterogeneous, multi-phase nature of the ISM. Improving over existing models, we compute a radiatively stable initial solution to avoid the transient collapse seen in models applying an isothermal stratification. The central feature of our simulations is the driving of turbulence via several thousand localized injections of thermal energy, which well resemble the kinetics of the supernova feedback. Unlike for artificial forcing, the energy and distribution of the SNe are determined by observable parameters.

In a preceding paper, we have shown that the turbulence created by SNe does in fact exponentially amplify the mean magnetic field (Gressel et al. 2008b). Here we extend this work towards a broader parameter base. The organisation of this article is as follows: in Section 1, we discuss the possible relevance of the discovered effect for realistic Reynolds numbers and compare the kinetically driven SN dynamo with the cosmic ray dynamo found by Hanasz et al. (2004). In Section 2, we then report on the influence of the supernova rate on the measured dynamo parameters. For a short review on mean-field modelling of the galactic dynamo, we refer the reader to Elstner et al. (this volume).

\section{Slow versus fast dynamo}

In laminar dynamos, diffusion sets the relevant timescale for magnetic reconnection, thus defining an upper limit for the allowable growth rate of the mean magnetic field. Because the microscopic diffusivity is usually low, these dynamos are commonly referred to as "slow dynamos". Within the ISM, the diffusion time $\tau_{\mathrm{d}}=L^{2} / \eta$ (related to the microscopic value $\eta \simeq 10^{8} \mathrm{~cm}^{2} \mathrm{~s}^{-1}$ ) by far exceeds the Hubble time. This means that the field amplification mechanism in galaxies needs to be a "fast dynamo" in the sense 

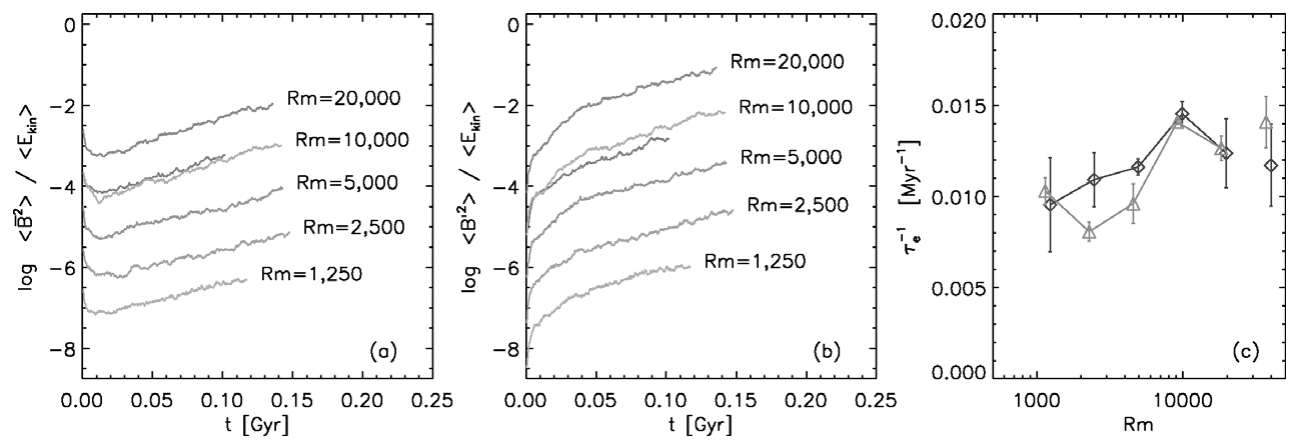

Figure 1. Evolution of the regular (a) and fluctuating (b) magnetic field strength for different Rm. For clarity, the ordinates of the different models have been offset by an order of magnitude each. In panel (c), we compare the growth rates for the turbulent (diamonds) and regular (triangles) magnetic field. The unconnected data points to the right correspond to a run with $\eta=0$ and provide an indication for the level of numerical diffusivity.

that it works on a timescale different than $\tau_{\mathrm{d}}$ (Lazarian \& Vishniac 1999). Accordingly, one assumes that the galactic dynamo is determined by some sort of effective turbulent diffusivity $\eta_{\mathrm{t}}$. Although the dynamo may still be limited by topological changes via reconnection, this process can be considerably faster due to the much higher value of $\eta_{\mathrm{t}}$ compared to $\eta$. Turbulent reconnection has recently been studied numerically by Otmianowska-Mazur, Kowal \& Lazarian (this volume).

According to the definition of $\tau_{\mathrm{d}}$, in the laminar case one expects the dynamo growth rate to increase with $\eta$. The picture, however, changes significantly as soon as the Reynolds number is high enough to allow for developed turbulence. Structures of integral length scale $L$ are now efficiently broken down to the Kolmogorov microscale where the atomic diffusion takes over. Varying the microscopic value of $\eta$ does only change the extent of the inertial range towards higher wavenumbers but is no longer relevant to the large-scale flow. This implies that a turbulent dynamo should be insensitive to variations in $\eta$ as soon as a critical value $\mathrm{Rm}_{\mathrm{c}}$ is exceeded (cf. Lazarian \& Vishniac 1999).

In Fig. 1, we plot the evolution of the mean and turbulent magnetic field (normalized to the kinetic energy) for different magnetic Reynolds numbers $\mathrm{Rm}=\mathrm{L}^{2} \mathrm{q} \Omega / \eta$. The values for $\mathrm{Rm}$ are obtained by varying $\eta$ while keeping the rotation rate $\Omega$ fixed. The kinematic viscosity $\nu$ is furthermore adopted to keep the magnetic Prandtl number $\mathrm{Pm}=\nu / \eta=2.5$ constant. Irrespective of the value of Rm, we observe a nice and steady exponential amplification of both the regular and turbulent fields. To estimate the influence of the finite grid resolution, we have performed a fiducial run (dark grey lines in panels (a) and (b) of Fig. 1) at double the grid spacing for $\mathrm{Rm}=10,000$. The obtained values are consistent with the higher resolved run and provide a first indication that the simulation results are reasonably converged at this level of dissipation and below.

In panel (c) of Fig. 1, we compare the growth rates for the turbulent (diamonds) and regular (triangles) magnetic field as a function of the magnetic Reynolds number. The unconnected data points to the right correspond to a run with $\eta=0$, i.e., the case of (formally) infinite Rm. As can be seen from a comparison with these points, above $\mathrm{Rm} \simeq 10,000$ we are limited by the finite value of the inherent numerical diffusivity of our code, i.e., better resolved runs become mandatory to study the regime of higher Rm. With respect to the reliably converged runs, we observe growth rates that increase with $\mathrm{Rm}$ - suggesting that the effect is of genuinely turbulent nature.

In their models of the cosmic-ray-driven buoyant instability, Otmianowska-Mazur et al. (2007) observe that their dynamo crucially relies on the presence of a "microscopic" 

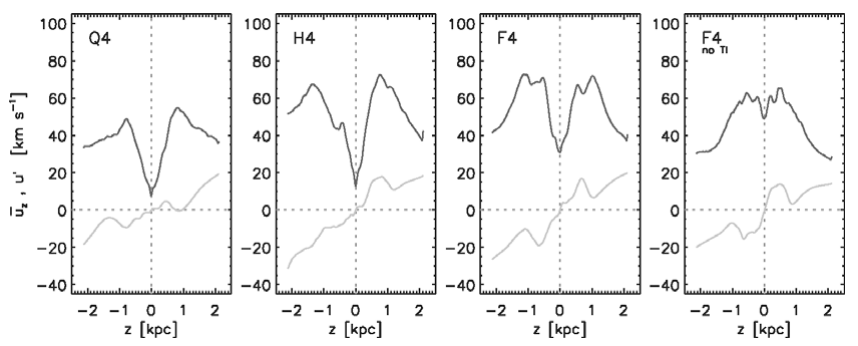

Figure 2. Vertical profiles of the mean flow $\bar{u}_{z}$ (light) and the turbulent velocity $u^{\prime}$ (dark colour) at quarter, half, and full supernova rate $\sigma / \sigma_{0}$.

diffusivity $\eta$. The authors actually find the efficiency of the field amplification to scale with this parameter. Moreover, they state that no scale separation is manifest in the spectra of their simulations. In this respect, it remains disputable whether the high value for $\eta$ might rather be interpreted as an effective turbulent dissipation $\eta_{\mathrm{t}}$. If so, the simulations would have to be regarded as large eddy simulations, i.e., simulations assuming turbulent effects rather than having them emerge from first principles.

\section{The dependence on supernova rate}

Quasilinear theory (Krause \& Rädler 1980) is a powerful tool in predicting the dynamo $\alpha$ effect from the underlying kinetic structure of the turbulent flow. Unfortunately, as far as galaxies are concerned, little has been known with certainty about the vertical profiles of the turbulent velocity $u^{\prime}(z)$ and the mean flow $\bar{u}_{z}(z)$. In particular, $\bar{u}_{z}$ had to be ignored in analytical derivations (see e.g. Fröhlich \& Schultz 1996) assuming hydrostatic equilibrium. In that regard, direct simulations can give important new insights, especially if one is interested in the dependence on factors like the supernova frequency.

\subsection{Vertical structure $\&$ wind}

In the following, we measure the supernova rate $\sigma$ in units of the galactic value $\sigma_{0}=$ $30 \mathrm{Myr}^{-1} \mathrm{kpc}^{-2}$, representative of type-II SNe. All models include sheared galactic rotation with $|q \Omega|=100 \mathrm{~km} \mathrm{~s}^{-1} \mathrm{kpc}^{-1}$. The time averaged turbulent and mean velocity profiles of three models with $\sigma / \sigma_{0}=0.25,0.5$, and 1.0 are depicted in Fig. 2: The vertical structure of the turbulent velocity $u^{\prime}$ shows a distinct M-shape, which peaks at $\pm 1 \mathrm{kpc}$. The positive gradient of $u^{\prime}$ in the central disk strongly suggests an inward transport of the mean magnetic field. The inner part of the profiles is similarly shaped as the ones obtained from MRI turbulence (Dziourkevitch et al. 2004; Piontek \& Ostriker 2007) but considerably steeper. Crudely extrapolating the fall-off in $u^{\prime}(z)$, we estimate that the MRI might become important in maintaining the observed velocity dispersions above galactic heights of $|z| \simeq 3 \mathrm{kpc}$.

While the overall amplitude of the turbulence increases with the SN intensity, its gradient is only weakly affected. This can be seen in Fig. 3, where we plot the scaling of the fitted velocity gradients. Unlike the turbulent velocity gradient $\mathrm{d} u^{\prime} / \mathrm{d} z$, which goes into saturation for $\sigma \simeq 0.1 \sigma_{0}$, the wind profile shows a distinct scaling with the supernova frequency. We thus estimate the wind profile from kinetic feedback as

$$
\bar{u}_{z}(z) \simeq 15 . \mathrm{km} \mathrm{s}^{-1}\left(\frac{\sigma}{\sigma_{0}}\right)^{0.4} \frac{\mathrm{z}}{1 . \mathrm{kpc}},
$$

which, of course, neglects the characteristic modulation of the mean flow within the $\mathrm{V}$-shaped region of $u^{\prime}(z)$, where the kinetic pressure counteracts the thermal pressure. 


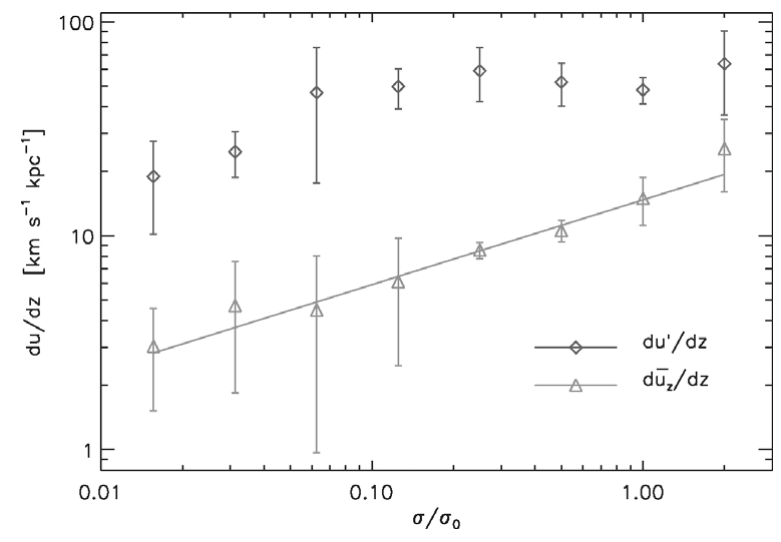

Figure 3. Vertical velocity gradients as a function of the supernova rate $\sigma / \sigma_{0}$. The values are derived from the inner disk for $u^{\prime}$, and from the full domain for $\bar{u}_{z}$ (cf. Fig. 2); the overplotted regression shows a logarithmic slope of 0.4 .
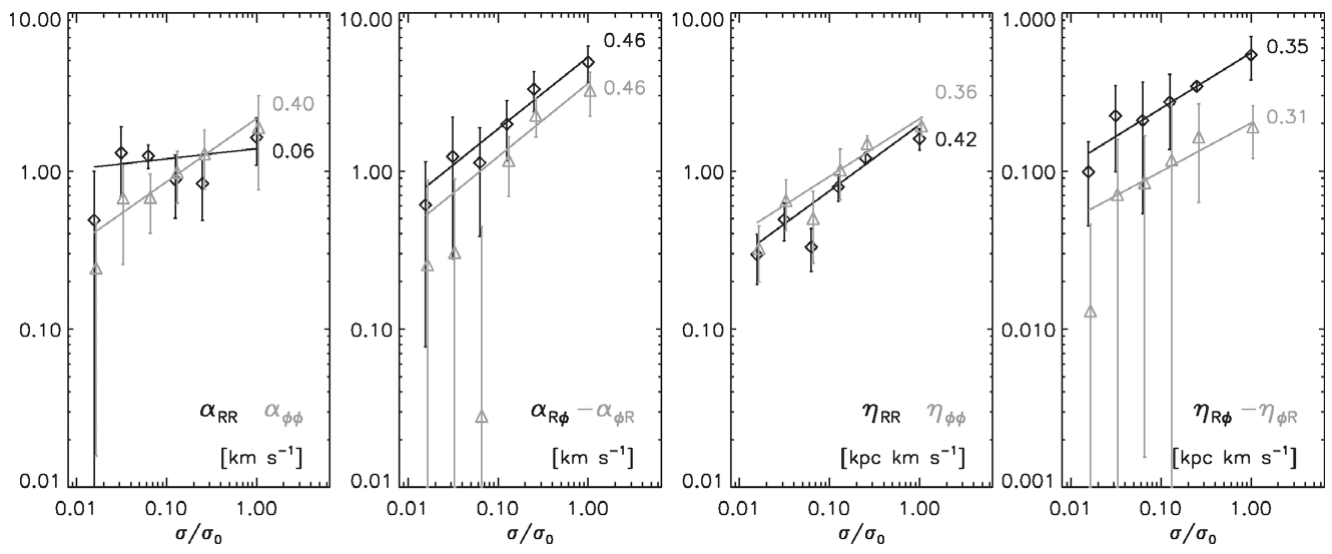

Figure 4. Coefficients of the dynamo $\alpha$ and $\tilde{\eta}$ tensor as obtained with the test-field method.

\subsection{Dynamo tensors \& growth rates}

One main focus of our work is the derivation of mean-field closure parameters from direct simulations. We here assume a standard parameterisation of the turbulent EMF

$$
\mathcal{E}_{i}=\alpha_{i j} \bar{B}_{j}-\tilde{\eta}_{i j} \varepsilon_{j k l} \partial_{k} \bar{B}_{l}, \quad i, j \in\{R, \phi\}, k=z,
$$

which enters the mean-field induction equation via $\nabla \times \mathcal{E}$. To measure these coefficients, we apply the test-field method of Schrinner et al. $(2005,2007)$. From the thus derived profiles $\alpha(z)$ and $\tilde{\eta}(z)$, we compute vertically averaged amplitudes. In Fig. 4, we plot these integral mean values as a function of the supernova rate $\sigma$. Notably, for each of the data points we had to perform a separate 3D simulation, covering a few hundred million years to obtain reasonable statistics. Despite the strong scatter in the plotted values, we observe a quite robust scaling with respect to $\sigma$. An exception to this are the coefficients $\alpha_{R R}$, which display irregular scaling in some of the runs and will be subject to further investigations.

The main result from Fig. 4 is that all coefficients (except $\alpha_{R R}$ ) scale in a similar way. In particular, this implies that the dynamo number

$$
C_{\alpha}=\alpha_{\phi \phi} H / \eta_{\mathrm{t}},
$$

remains approximately constant with $\sigma$. Furthermore, as we have already demonstrated in an earlier work, the effects of the off-diagonal elements $\alpha_{R \phi}$ and $\alpha_{\phi R}$ (which are responsible for the diamagnetic pumping) are approximately balanced by the mean flow 


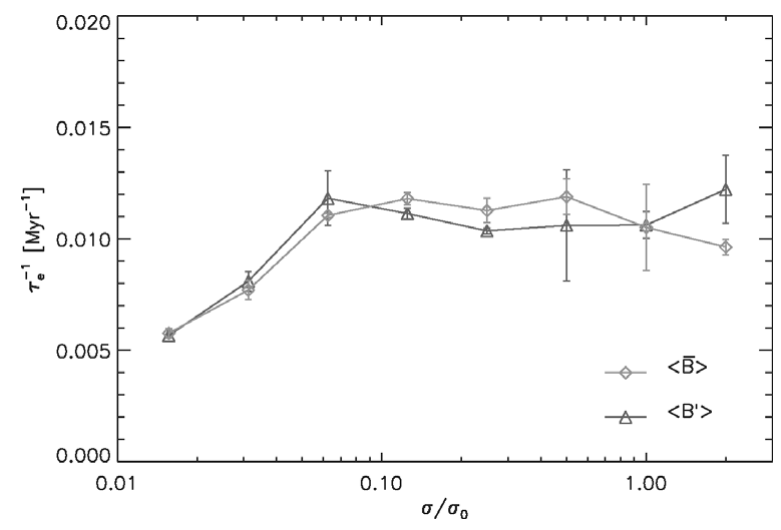

Figure 5. Growth rate $\tau_{\mathrm{e}}^{-1}$ of the (vertically integrated) regular field $\langle\bar{B}\rangle$ (diamonds) and the turbulent field $\left\langle B^{\prime}\right\rangle$ (triangles) as a function of $\sigma / \sigma_{0}$.

$\bar{u}_{z}$. Comparing the slope shown in Fig. 3 with the ones in the second panel of Fig 4, we see that this balance of forces is roughly independent of $\sigma$ and therefore seems to be of rather general nature.

With the constant ratio of the $\alpha$ effect versus the turbulent diffusion $\eta_{\mathrm{t}}$ on one hand, and the mutual balance of the turbulent pumping and mean flow on the other hand, we expect the overall growth rates to be largely insensitive to the applied supernova rate. For sufficiently high values of $\sigma$ this is indeed the case, as can be seen from Fig. 5 above - we want to point out that these growth rates are obtained from the direct simulations and hence do not depend on the $\alpha$ prescription. With constant growth rates over more than one order of magnitude in star formation activity, the kinetically driven SN dynamo proves to be rather universal. Accordingly, it may well be applicable to a wide range of scenarios - covering dwarf galaxies as well as starbursts.

\section{Conclusions}

We have presented recent simulation results on the variation of the key dynamo parameters with the supernova frequency. Yet more work is needed to understand how the vertical structure of the galactic disk is linked to the emergence of the dynamo effect and the associated transport processes. As a primary question, one is of course interested in finding a simple explanation for the observed scaling relations - which are somewhat flatter than the square-root of the supernova rate $\sigma$.

From quasilinear theory, it is understood that the dynamo effect depends on vertical gradients both in the density $\rho$ and the turbulence intensity $u^{\prime}$. The disk structure, on the other hand, is determined by the balance of the kinetic pressure from the forcing and the gravitation of the stellar component - here equipartition arguments can yield a first approximation. To connect kinematic properties with dynamo parameters, we however need to specify the correlation time $\tau_{\mathrm{c}}$ of the turbulent flow. By comparing direct simulations with SOCA predictions, it will eventually become possible to determine how this quantity depends on the supernova frequency.

To connect the measured dynamo parameters $\alpha$ and $\tilde{\eta}$ with the growth rates observed in the simulations - and thereby check the applicability of the approach - more meanfield modelling is necessary. We are, however, confident that the classical framework of MF-MHD remains a valuable tool in the quest of understanding galactic magnetism.

\section{Acknowledgements}

This project was kindly supported by the Deutsche Forschungsgemeinschaft (DFG) under grant $\mathrm{Zi}-717 / 2-2$. All computations were performed at the AIP babel cluster. 


\section{References}

Dziourkevitch, N., Elstner, D., \& Rüdiger, G. 2004, A\& $A$ 423, L29

Fröhlich, H.-E. \& Schultz, M. 1996, A\&BA 311, 451

Gressel, O., Ziegler, U., Elstner, D., \& Rüdiger, G. 2008a, $A N$ 329, 619

Gressel, O., Elstner, D., Ziegler, U., \& Rüdiger, G. 2008b, A\& A 486, L35

Hanasz, M., Kowal, G., Otmianowska-Mazur, K., \& Lesch, H. 2004, ApJ 605, L33

Krause, F. \& Rädler, K. H. 1980, Mean-field magnetohydrodynamics and dynamo theory (Oxford: Pergamon Press)

Lazarian, A. \& Vishniac, E. T. 1999, ApJ 517, 700

Otmianowska-Mazur, K., Kowal, G., \& Hanasz, M. 2007, ApJ 668, 110

Piontek, R. A. \& Ostriker, E. C. 2007, ApJ 663, 183

Schrinner, M., Rädler, K.-H., Schmitt, D., Rheinhardt, M., \& Christensen, U. 2005, AN 326, 245

Schrinner, M., Rädler, K.-H., Schmitt, D., Rheinhardt, M., \& Christensen, U. R. 2007, GApFD 101,81

\section{Discussion}

Johansen: Do you see any signs of Parker instability in the azimuthal fields?

Gressel: Typically, our simulations are seeded with dynamically weak magnetic fields $\left(\beta_{\mathrm{P}}=2 \times 10^{7}\right)$. If we, however, start with stronger fields and approach equipartition strengths, we clearly see buoyancy effects.

De Gouveia dal Pino: 1) You have obtained the appropriate B fields through a SNdriven dynamo but what were the coherence scales? 2) How could you compare your 3D MHD galaxy SN-wind model with those by Avillez and colleagues?

Gressel: 1) From the inferred velocity structure functions, we derive a coherence-length of 80-100 pc, which coincides with the outer scale of SNRs but is considerably smaller than the typical sizes of super-bubbles. The scale of the observed mean field is the scaleheight of the inner thick disk, which is of the same order. 2) The models are very similar. However, we include galactic rotation and shear, which are not present in their models and are a mandatory prerequisite for the excitation of a mean-field dynamo.

BRANDENBURG: I agree with you that shear also produced an $\alpha$ effect, but it has the opposite effect than the effect caused by rotation; although it is not as strong. What seems to matter is $2 \Omega+\overline{\mathbf{W}}$, with $\overline{\mathbf{W}}=\nabla \times \overline{\mathbf{U}}$ is the vorticity of the shear flow. As you say, the Parker instability becomes important for strong magnetic fields, and so does the MRI. In that case $\alpha$ really reverses sign and should be proportional to $\overline{b_{x} b_{y}}$. Would you agree?

Gressel: The $\alpha$ effect caused by shear is only somewhat weaker than the effect caused by the rotation. Otherwise I agree with your statement. 